\title{
Gut microbiota response to antibiotics is personalized and depends on baseline microbiota
}

\author{
Armin Rashidi ${ }^{1 *} \mathbb{D}$, Maryam Ebadi ${ }^{1}$, Tauseef Ur Rehman² ${ }^{2}$,Heba Elhusseini ${ }^{1}$, Harika Nalluri ${ }^{3}$, Thomas Kaiser ${ }^{3}$, \\ Shernan G. Holtan ${ }^{1}$, Alexander Khoruts ${ }^{2}$, Daniel J. Weisdorf ${ }^{1}$ and Christopher Staley ${ }^{3}$
}

\begin{abstract}
Background: The magnitude of microbiota perturbations after exposure to antibiotics varies among individuals. It has been suggested that the composition of pre-treatment microbiota underpins personalized responses to antibiotics. However, this hypothesis has not been directly tested in humans. In this high-throughput amplicon study, we analyzed 165 ribosomal RNA gene sequences of 260 stool samples collected twice weekly from 39 patients with acute leukemia during their $\sim 4$ weeks of hospitalization for chemotherapy while they received multiple antibiotics.
\end{abstract}

Results: Despite heavy and sustained antibiotic pressure, microbial communities in samples from the same patient remained more similar to one another than to those from other patients. Principal component mixed effect regression using microbiota and granular antibiotic exposure data showed that microbiota departures from baseline depend on the composition of the pre-treatment microbiota. Penalized generalized estimating equations identified 6 taxa within pre-treatment microbiota that predicted the extent of antibiotic-induced perturbations.

Conclusions: Our results indicate that specific species in pre-treatment microbiota determine personalized microbiota responses to antibiotics in humans. Thus, precision interventions targeting pre-treatment microbiota may prevent antibiotic-induced dysbiosis and its adverse clinical consequences.

Keywords: Antibiotics, Leukemia, Microbiota

\section{Background}

Antibiotics represent a major cause of perturbation experienced by microbial communities in the body. The typical progression of the compositional states of microbiota after antibiotic-induced perturbations includes departure from baseline (phase 1) followed by post-antibiotic reorganization (phase 2) (Fig. 1). Reorganization of microbiota composition after cessation of antibiotics may lead to full recovery to the

\footnotetext{
* Correspondence: arashidi@umn.edu

1 Division of Hematology, Oncology, and Transplantation, Department of Medicine, University of Minnesota, 14-100 PWB, MMC 480, 420 Delaware St. SE, Minneapolis, MN 55455, USA

Full list of author information is available at the end of the article
}

original pre-treatment state. Alternatively, recovery may be partial, with the new community having some degree of similarity to pre-treatment microbiota. Yet in other cases, reorganizing microbiota can shift to completely new states with little resemblance to the baseline community [1-3], commonly referred to as a "regime shift" $[4,5]$. A resistant community can be defined as one that resists perturbation, while a resilient community is one that recovers after perturbation [6]. A resilient community will likely stabilize into a fully functional state after transitioning through phases of perturbation and recovery $[7,8]$. Unresolved disruptions in microbiota lead to loss of colonization resistance [9], deleterious health

C C The Author(s). 2021 Open Access This article is licensed under a Creative Commons Attribution 4.0 International License, which permits use, sharing, adaptation, distribution and reproduction in any medium or format, as long as you give appropriate credit to the original author(s) and the source, provide a link to the Creative Commons licence, and indicate if changes were made. The images or other third party material in this article are included in the article's Creative Commons licence, unless indicated otherwise in a credit line to the material. If material is not included in the article's Creative Commons licence and your intended use is not permitted by statutory regulation or exceeds the permitted use, you will need to obtain permission directly from the copyright holder. To view a copy of this licence, visit http://creativecommons.org/licenses/by/4.0/ The Creative Commons Public Domain Dedication waiver (http://creativecommons.org/publicdomain/zero/1.0/) applies to the data made available in this article, unless otherwise stated in a credit line to the data. 


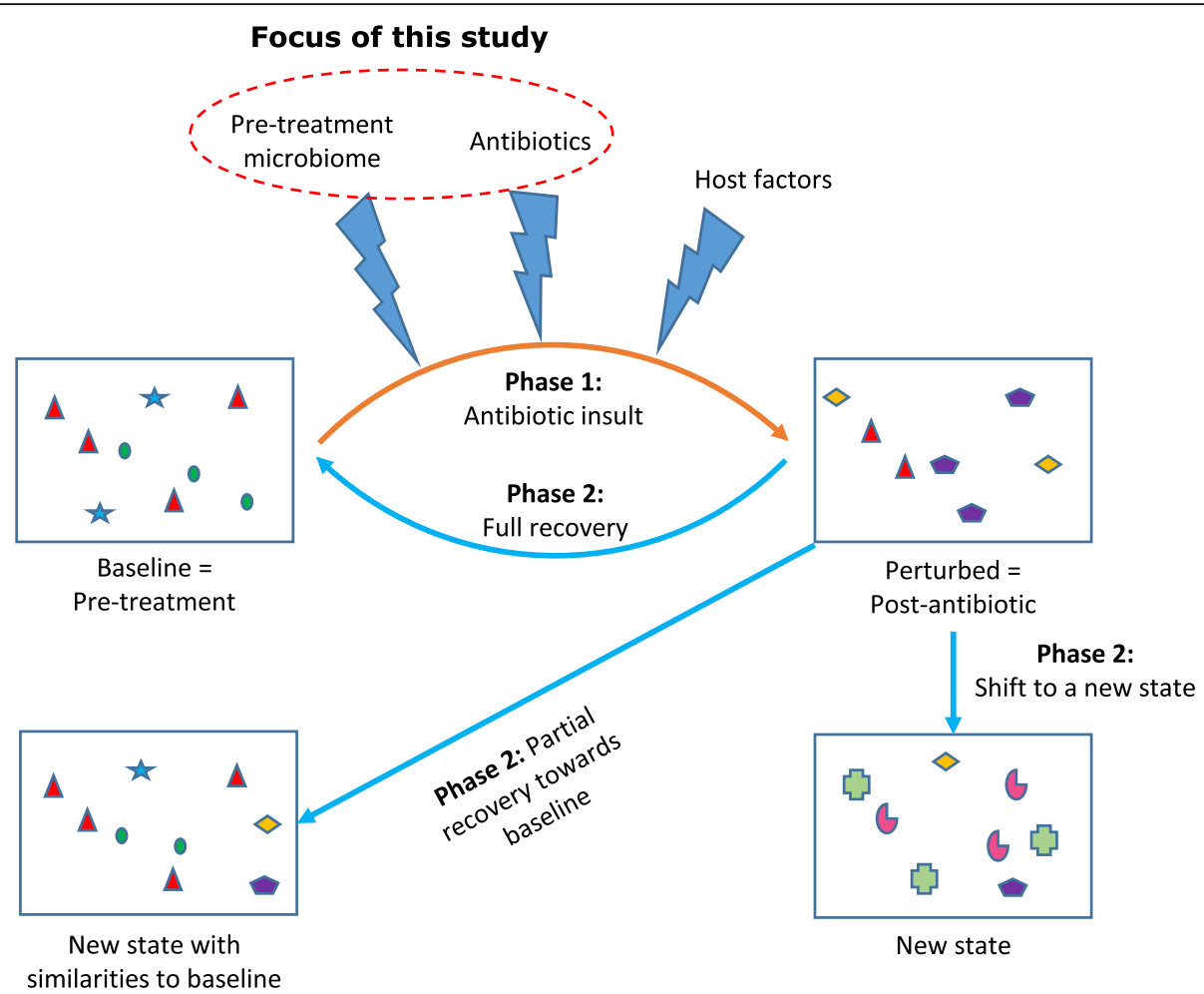

Fig. 1 Phases of microbiota response to antibiotics. After antibiotic exposure, baseline microbiota undergoes an initial perturbation (phase 1), during which some taxa may completely disappear, while some new taxa may arise. Potential determinants of phase 1 include baseline microbiota, specifics of antibiotic exposure, and host factors (e.g., genetics and immunity). After completion of antibiotic treatment, the microbiota undergoes reorganization (phase 2), which could lead to full recovery to baseline, partial recovery towards baseline, or shift to a completely new state. The focus of this study is the role of baseline microbiota in regulating phase 1 after controlling for antibiotic exposures

consequences such as Clostridioides difficile infection [10], and altered metabolic functions important for host food processing [11], drug metabolism [12], and endocrine homeostasis (e.g., appetite regulation) [13].

Microbiota response to antibiotics varies among individuals [2] and the mechanism of response is poorly understood [1, 14]. Intuitively, characteristics of the antibiotic insult (e.g., antibiotic type and duration), composition and wiring of pre-treatment microbiota, and host factors (e.g., genetics and immunity [15]) are possible determinants of individualized microbiota responses. Here, we hypothesize that pre-treatment microbiota in humans is a determinant of community resistance as measured inversely by the magnitude of microbiota deviation from baseline during phase 1. Pre-clinical data support this hypothesis. Germ-free mice humanized with fecal microbiota from different donors show different microbiota responses to the same antibiotic [16], suggesting that pre-treatment microbiota may underpin individualized responses. In addition, the bloom of specific pathogens after antibiotic treatment has been associated with the initial state of the microbiota [17]. However, it is unknown whether and to what extent specific species in baseline microbiota modulate community resistance against antibiotic perturbation.

Antibiotic exposure may be light and brief (e.g., one dose of one antibiotic), light and sustained (e.g., severalday course of one antibiotic), heavy and brief (e.g., brief course of multiple antibiotics), or heavy and sustained (e.g., prolonged course of multiple antibiotics). We studied patients with acute myeloid leukemia (AML) receiving inpatient chemotherapy, a prototype setting for heavy sustained antibiotic exposure. These patients receive multiple antibiotics over several weeks of hospitalization. Initially, patients receive antibiotics to prevent infections. However, most patients develop a fever despite prophylaxis [18]. At the time of fever, prophylactic antibiotics are escalated to broader-spectrum antibiotics, which are later adjusted according to the results of microbiological work-up. These antibiotics are de-escalated to prophylactic antibiotics or discontinued as clinical and hematopoietic recovery ensue. This cascade of events can be summarized as overlapping waves of antibiotic exposure, sometimes including multiple antibiotics for extended periods. 
High-throughput amplicon sequencing of longitudinal stool samples collected from hospitalized patients with AML coupled with granular antibiotic exposure data uniquely positioned us to measure the dependency of microbiota perturbations on the microbiota's baseline state. We find that the composition of pre-treatment microbiota is a major determinant of the magnitude of microbiota departure from baseline. We identify pretreatment taxa that stabilize or destabilize the microbiota. These findings offer a mechanistic explanation for individualized responses to antibiotics and introduce novel targets for precision interventions to prevent antibiotic-induced dysbiosis and its adverse clinical consequences.

\section{Methods}

\section{Patients and samples}

We enrolled hospitalized adult patients with newly diagnosed or relapsed/refractory AML to a prospective, biorepository protocol (registration number in ClinicalTrials. gov: NCT03316456) approved by the University of Minnesota Institutional Review Board. An expected $\sim 4$ weeks of hospitalization was required. No other inclusion or exclusion criteria were used. Clinical and supportive care followed our standard institutional algorithm. Deviations per the treating physicians' discretion were permitted. Our antibiotic stewardship recommends acyclovir for viral, an azole for fungal, and levofloxacin for bacterial prophylaxis for the duration of neutropenia. Bacterial prophylaxis is continued until development of neutropenic fever or first neutrophil rise above $1 \times 10^{9} / \mathrm{L}$, whichever occurs first. Cefepime is our recommended empiric frontline antibiotic for neutropenic fever. When oral intake decreases to $<60 \%$ of the lower limit of estimated energy and protein needs for 7 days, we generally initiate parenteral nutrition.

Stool samples were collected twice weekly (Mondays and Thursdays, \pm 1 day window) between hospital admission and day 28 of chemotherapy or discharge (whichever occurred first). Collections were independent of clinical factors. Stool samples were collected in 95\% ethanol-filled sterile tubes and stored at $-80{ }^{\circ} \mathrm{C}$. Antibiotic exposure data were collected from electronic medical records for the following classes of antibacterial antibiotics: fluoroquinolones, 3rd or higher generation cephalosporins, piperacillin-tazobactam, carbapenems, metronidazole, oral vancomycin, and intravenous vancomycin.

\section{$16 \mathrm{~S}$ ribosomal RNA ( $\mathrm{RNNA}$ ) gene sequencing}

DNA was extracted using the DNeasy PowerSoil DNA isolation kit (QIAGEN, Hilden, Germany). The V4 hypervariable region of the $16 \mathrm{~S}$ rRNA gene was amplified on an Illumina MiSeq platform $(2 \times 300$ paired-end mode) by the University of Minnesota Genomics Center [19]. Sequences were processed in QIIME 2 [20]. Quality filtering, adaptor trimming, and stitching of raw sequences were done using the quality control pipeline SHI7 (trim threshold 32, threshold of Q37) [21]. Paired ends were merged using FLASH [22]. Operational taxonomic unit (OTU) picking was done using NINJA-OPS (default parameters and 97\% similarity threshold) and the Greengenes database; Bowtie 2 was used for alignment [23-25]. Species-level taxonomy was not considered given our short amplicon methodology. OTUs with a frequency of $<0.01 \%$ of reads and samples with fewer than 500 reads were removed. Sequence data were deposited in the Sequence Read Archive of the National Center for Biotechnology's Information (NCBI) under BioProject ID SRP141394. The BIOM table was exported from QIIME into R 3.4 (R Foundation for Statistical Computing, Vienna, Austria).

\section{Microfluidic quantitative PCR for antibiotic resistance genes}

Remaining DNA from a subset of samples was used for microfluidic qPCR (MFQPCR) to quantify various antibiotic-resistance genes (ARGs) including those for beta-lactams, quinolones, and vancomycin resistance as previously described [26]. BioMark HD System (Fluidigm) and 96.96 Dynamic Array IFCs (Fluidigm) were used for analysis. Serial dilutions $\left(2 \times 10^{0}\right.$ to 2 $\times 10^{6}$ copies $/ \mu \mathrm{L}$ ) of the mixture of synthetic DNA fragments containing each target gene sequence were also included in the analysis to generate standard curves. Specific target amplification (STA) was done with 14 PCR cycles to increase the target DNA molecule prior to MFQPCR as previously described [27]. Sample DNA, gBlock standard mixtures, and no template controls were subjected to STA. Quantitative results obtained by MFQPCR were analyzed using RealTime PCR Analysis software version 4.1.2 (Fluidigm) as described previously [27]. All samples were run in duplicate and their average gene content for each ARG (gene copies/microL DNA) was calculated.

\section{Statistical analysis}

All analyses were performed using custom scripts and packages phyloseq, vegan, lme4, and PGEE in R. Linear discriminant analysis (LDA) coupled with effect size measurements (LEfSe) using patient number as "subject" was used to find taxa that differed significantly (LDA score $>3.0, p<0.05)$ between the two groups [28]. OTU abundances were centered log-ratio (clr) transformed to account for the compositionality of microbiota. Dissimilarity between samples was quantified by Aitchison distance [29]. A pseudocount of $\mathrm{min} / 2$ was added to exact zeros prior to transformation. Alpha diversity was 
estimated by Shannon's index [30]. We used $\Delta \mathrm{m}$ to denote Aitchison distance between non-baseline and baseline (pre-treatment) microbiota for the same patient, providing a single numerical value representing the magnitude of microbiota perturbation from baseline. Ordination was visualized by principal component analysis (PCA) and the proportion of variance explained by the individual was determined by permutational analysis of variance (PERMANOVA) with an adonis test (999 permutations) [31]. Other variables included in adonis were the first PCA axis of antibiotic history (see below), use of parenteral nutrition, and interval from baseline. Samples were the units of analysis and $\Delta \mathrm{m}$ was the outcome variable in all models unless specified otherwise. The performance of all models was estimated by Pearson's correlation coefficient comparing observed vs. predicted values of $\Delta \mathrm{m}$.

\section{Quantification of antibiotic history}

We quantified the "antibiotic history" of each sample using the time series of exposures to 7 major classes of antibacterial antibiotics between hospital admission and the day the sample was collected. The antibiotic classes considered were fluoroquinolones, third or higher generation cephalosporins, metronidazole, piperacillin-tazobactam, intravenous vancomycin (also daptomycin or linezolid), oral vancomycin, and carbapenems. For a given day, if a given antibiotic was used, it was coded 1 and otherwise, zero. Day 0 was defined as the first day of chemotherapy. Next, we applied a decaying average function to the time series of $0 \mathrm{~s}$ and $1 \mathrm{~s}$ for each antibiotic class to achieve a single numerical value summarizing exposure history for the given sample and antibiotic. As an example, if levofloxacin was used on days 1-3 for a patient admitted on day -1 ( 1 day before starting chemotherapy), the time series for levofloxacin for a sample collected on day 5 of chemotherapy from this patient would be $(0,0,1,1,1,0)$, indicating that the antibiotic was not used on days - 1 , 0 (first day of chemotherapy), and 4, but was used on days 1,2 , and 3 . With a decay factor of 2 , the levofloxacin history for this sample would be quantified and summarized as $0 \times$ $2^{0}+1 \times 2^{-1}+1 \times 2^{-2}+1 \times 2^{-3}+0 \times 2^{-4}+0 \times 2^{-5}$ $+0 \times 2^{-6}=0.875$. With this decay factor, exposure on a given day receives twice as much weight than exposure on the previous day. A smaller decay factor would make the differences between the weights smaller. We ran Procrustes analysis [32] (999 permutations) to evaluate the correlation between microbiota composition (Aitchison distance) and antibiotic history (Euclidean distance); the sensitivity of this correlation to the decay factor was assessed.

\section{Principal component linear mixed effect regression (package Ime4, function Imer)}

The goal of this model was to evaluate whether baseline microbiota predicts $\Delta \mathrm{m}$ after controlling for antibiotics and other covariates. Independent fixed effect variables included the following: the top principal components (PCs) of antibiotic history for each non-baseline sample, the top microbiota PCs for baseline microbiota, use of parenteral nutrition, interval from baseline, baseline Shannon index, and sample read depth. Patient ID was a random effect. $p$ values were estimated from 200 bootstraps.

\section{Penalized generalized estimating equations (PGEE) [33, 34]}

While principal component regression allows us to understand the association between baseline microbiota and $\Delta \mathrm{m}$ after adjusting for covariates, it does not identify specific pre-treatment taxa that made the largest contribution to $\Delta \mathrm{m}$. We implemented penalized GEE to analyze longitudinal data with many covariates [35]. The main idea of PGEE is that in models with many potential microbiota predictors, typically most taxa do not contribute to the outcome variable, and thus, regression coefficients for those variables should be zero. Using a penalty function, PGEE shrinks the estimates of small regression coefficients toward zero. A cutoff of $10^{-3}$ for shrunken coefficients is conventionally used to exclude variables from the model. Variables that remain in the final model are important predictors of the outcome variable, regardless of the specific value of their regression coefficients. The original model included 133 features: clr abundances of the OTUs collapsed at genus level (121 features) at baseline, decay averaged antibiotic histories (7 features representing 7 antibiotic classes), age, sex, body mass index, use of parenteral nutrition (categorical), baseline Shannon index, sample depth, and interval from baseline (days). Patient ID was a random effect. Fourfold cross validation (function CVfit) was used to estimate the optimal value of the tuning parameter, which was then used to build the final model (function PGEE). A first-order autoregressive correlation structure (i.e., observations closer to each other being more strongly correlated than those farther apart) was assumed. PGEE produces a consistent and asymptotically normal estimator even if the assumed correlation structure is misspecified. $p<0.05$ was used to define statistical significance.

\section{Results}

Thirty-nine patients with AML provided twice-weekly stool samples during their $\sim 4$ weeks of hospitalization for chemotherapy. Fifteen patients required parenteral nutrition, and all but one patient developed a fever. As expected, antibiotic exposure was heavy and sustained 


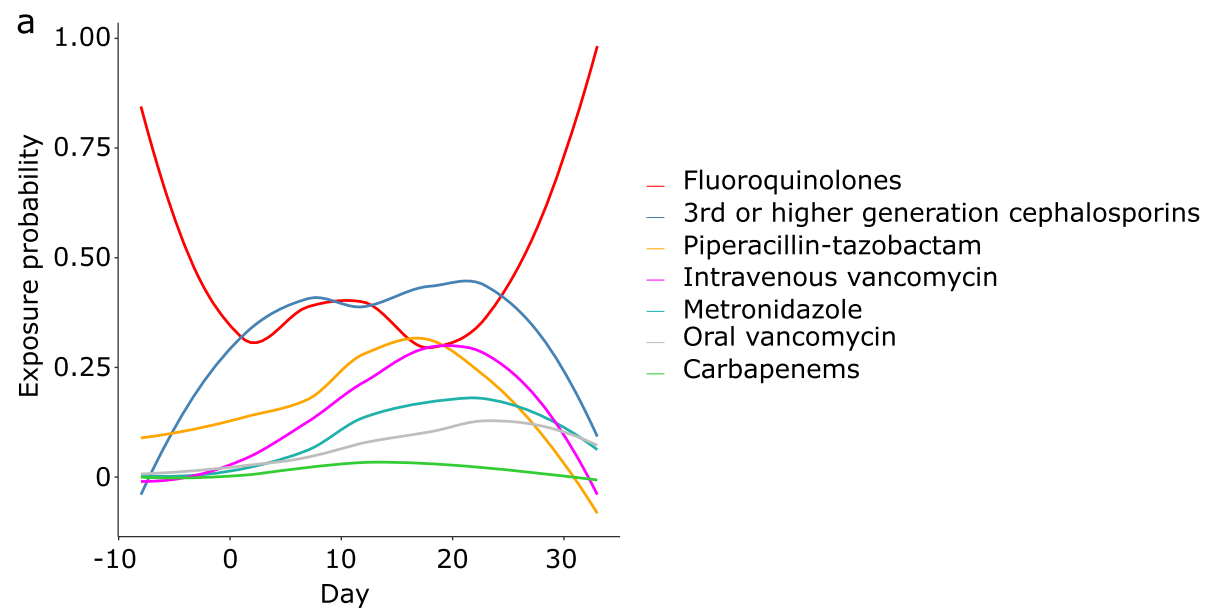

b
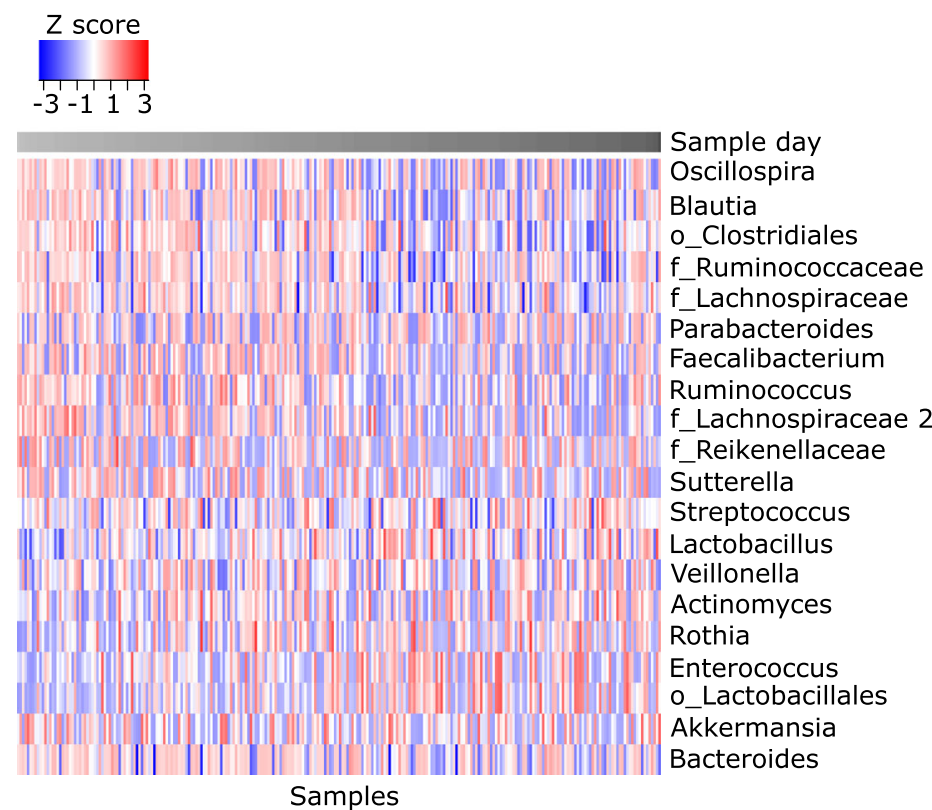

Fig. 2 Antibacterial antibiotic exposures and microbiota heatmap. a Probability of exposure to each class of antibacterial antibiotics over time, with time measured in days and relative to the first day of chemotherapy. Linezolid and daptomycin were grouped together with intravenous vancomycin. Exposure for a typical patient starts with fluoroquinolone prophylaxis, followed by neutropenic fever, when fluoroquinolone is changed to broader-spectrum antibiotics (especially cefepime, piperacillin-tazobactam, and vancomycin); hence, there is an initial decline in the fluoroquinolone curve, but rises in other antibiotic curves. Antibiotic pressure peaks in weeks 2 and 3. Concurrent with clinical and hematopoietic recovery in week 4, broad-spectrum antibiotics are discontinued or de-escalated to prophylactic fluoroquinolones, the latter explaining the late rise in the fluoroquinolone curve. $\mathbf{b}$ Heatmap of the 20 most abundant genera. Each column indicates a sample, and each row represents genuslevel mapping of an OTU. $f$ (family) and o (order) indicate OTUs that were unclassifiable to deeper levels. OTU relative abundances were Ztransformed (indicated by the color gradient). Rows were ordered by their mean values and columns by sample collection day (color bar at top) relative to the first day of chemotherapy, with lighter colors indicating earlier days

(Fig. 2a). After filtering, we analyzed 260 samples (median of 7 per patient) containing an average of 19,604 high-quality reads per sample. A heatmap of the 20 most abundant genera is shown in Fig. 2b. Additional file 1: Figure S1a shows the aggregate relative abundance of the most abundant taxa in baseline and non-baseline taxa. Additional file 1: Figure S1b shows differentially abundant taxa in each group, adjusting for patient ID. The genus most prominently expanding from baseline to subsequent samples was Enterococcus (Additional file 1: Figure S1), consistent with our previous reports [36, 37].

The microbiota became more dissimilar to baseline over time (Fig. 3a). Despite this progressive departure from baseline, the greatest proportion of microbiota variance was explained by the individual (PERMANOVA $R^{2}=0.54, p<0.001$, adonis test with 999 permutations; Fig. 3b). Although interval from baseline, use of parenteral nutrition, and antibiotic history were also 


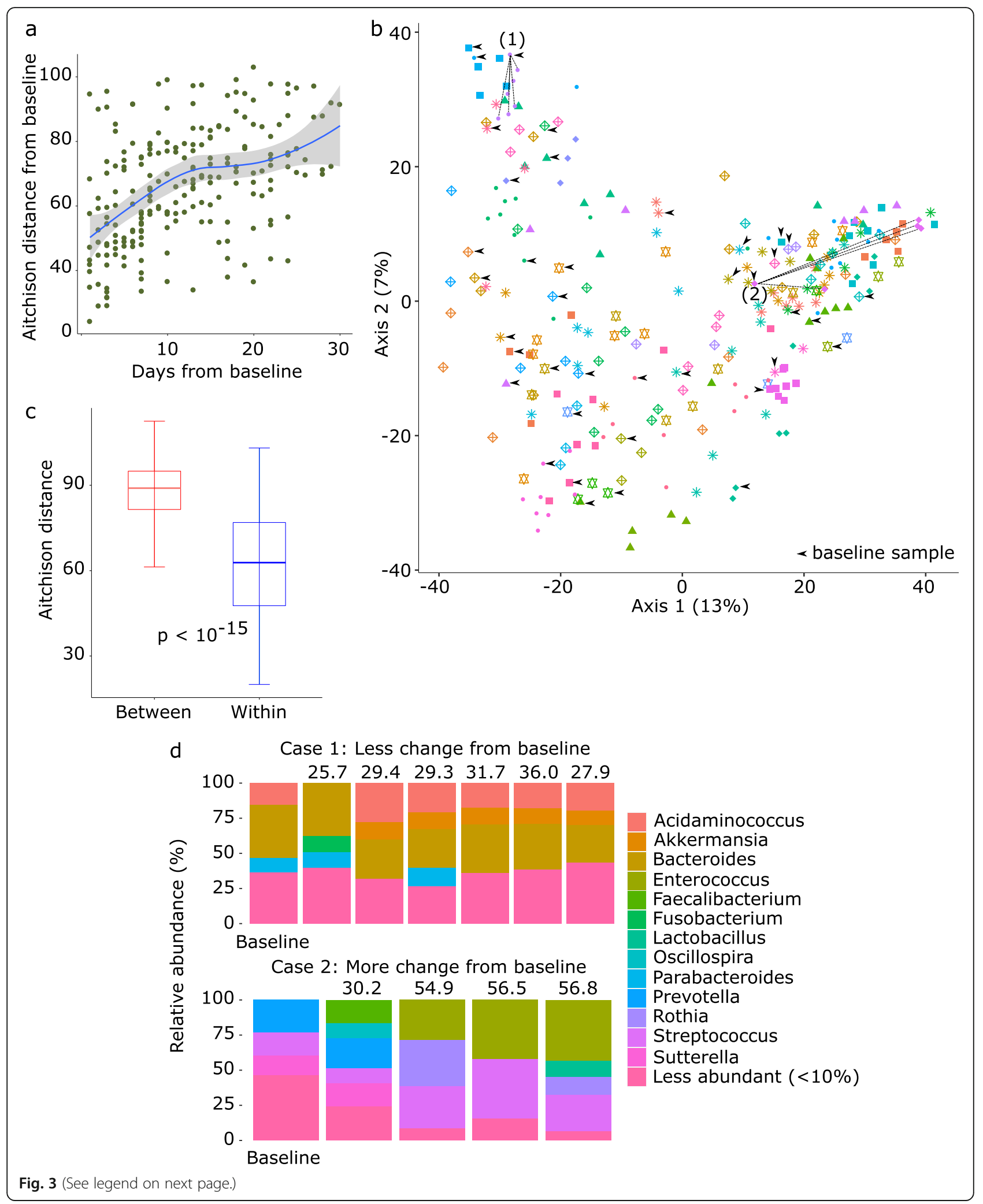


(See figure on previous page.)

Fig. 3 Microbiota variation among patients and over time. a Microbiota distance from baseline, as measured by Aitchison distance, vs. time. Time was measured in days relative to the baseline sample. The loess smoothed curve and its $95 \%$ confidence bar are shown. b Principal component analysis using Aitchison distance. Each point represents a sample, and points with the same color and shape represent samples from the same patient. Baseline samples are indicated by arrowheads. Numbers in parentheses indicate percent variation explained by the corresponding principal component. For two subjects (cases 1 and 2), non-baseline samples are connected to the baseline sample for the same patient. c Aitchison distance between samples collected from the same patient ("within") vs. different patients ("between"). $p$ value was calculated from a Wilcoxon test. $\mathbf{d}$ Distribution of taxa among the samples in the two cases (1 and 2) in $\mathbf{b}$. Genera with relative abundances $<10 \%$ across all samples are grouped together. Baseline sample from each patient is shown on the left. The number above each non-baseline sample indicates its Aitchison distance from the corresponding baseline sample

statistically significant in this analysis $(p<0.001)$, they only explained a small proportion of the variance (PERMANOVA $R^{2}=0.019,0.007$, and 0.006, respectively). Median Aitchison distance between samples from the same patient was significantly smaller than median distance between samples from different patients $(p<$ $10^{-15}$; Wilcoxon test; Fig. 3c), indicating that microbial communities in samples from the same patient remained more similar to one another than to those from the other patients. As a potential mechanism for this host specificity, we evaluated whether baseline microbiota was a determinant of subsequent microbiota departures from baseline after controlling for antibiotics. Microbiota departure from baseline was denoted $\Delta \mathrm{m}$ and defined as the Aitchison distance [29] between baseline and non-baseline communities. We first quantified the antibiotic history of each sample by considering the time series of exposures to 7 major classes of antibacterial antibiotics between hospital admission and day the sample was collected. For a given day, if a given antibiotic was used, exposure to that antibiotic was coded 1 and otherwise coded zero. Next, we applied a decaying average function to the antibiotic history of each sample-a time series of $0 \mathrm{~s}$ and $1 \mathrm{~s}$ for each antibiotic class-to achieve a single numerical value summarizing the exposure history of a given sample for a given antibiotic. The decaying average method flexibly models both recent and less recent exposures by placing more weight on exposures in more recent days preceding the sample. Procrustes analysis (Additional file 1: Figure S2) showed that the correlation between microbiota composition and antibiotic history was robust to the specific choice of the decay factor. We chose a decay factor of 2 for our main analysis thereafter.

Next, we used principal component mixed effect regression including the top principal components (PCs) of antibiotic history for each non-baseline sample and the top PCs of the microbiota for the baseline sample from the same individual as predictors of $\Delta \mathrm{m}$. This approach eliminates the problem of multicollinearity because the PCs are orthogonal. We included the first PC of the microbiota and the first $\mathrm{PC}$ of antibiotic history as predictors in the model. In addition, use of parenteral nutrition (categorical), interval from baseline (days), baseline Shannon index, and sample read depth were included as fixed effect covariates and patient ID as a random effect. The model was powerful, with a Pearson's correlation coefficient comparing observed vs. predicted $\Delta \mathrm{m}$ values of 0.82 (Additional file 1: Figure S3a). Microbiota and antibiotic PCs and interval from baseline were significant predictors of $\Delta \mathrm{m}$ (Table 1). Although including more microbiota PCs would explain a larger proportion of microbiota variance, it did not improve performance of the regression model and the correlation coefficient remained 0.82 . Model performance did not change when a slower decay (decay factor 1.1) was used to define antibiotic history (Additional file 1: Figure $\mathrm{S} 3 \mathrm{~b})$. These findings indicate that baseline microbiota is a major independent determinant of $\Delta \mathrm{m}$.

To identify pre-treatment taxa with the largest contribution to $\Delta \mathrm{m}$, we used the penalized generalized estimating equations (PGEE) method, which is suitable for longitudinal high-throughput data analysis [33, 34]. Features were baseline genus-level clr abundances (121

Table 1 Principal component mixed effect regression to evaluate whether baseline microbiota independently predicts $\Delta \mathrm{m}$

\begin{tabular}{llll}
\hline Variable & Regression coefficient & Standard error & P \\
\hline Baseline microbiota PC1 & 0.82 & 0.35 & 0.01 \\
Antibiotic PC1 & -1.90 & 0.77 & 0.01 \\
Interval from baseline (days) & 0.98 & 0.12 & $<0.01$ \\
Baseline Shannon index & -5.70 & 4.19 & 0.19 \\
Sample read depth & 0.88 & 1.96 & 0.54 \\
Parenteral nutrition & 3.60 & 3.38 & 0.37 \\
\hline
\end{tabular}

$P C$ principal component; $\Delta \mathrm{m}$, Aitchison distance between the baseline and subsequent microbiota 


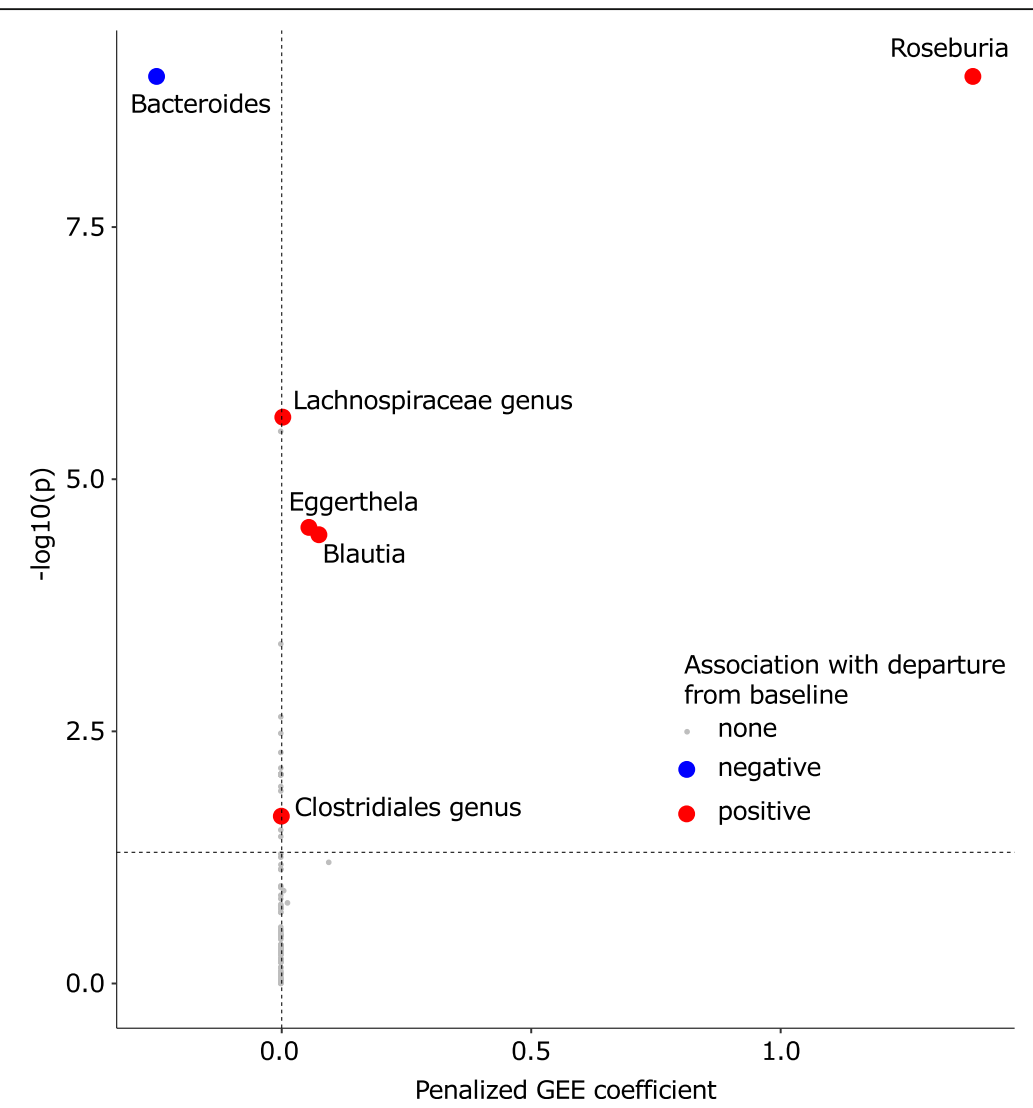

Fig. 4 Baseline taxonomic predictors of microbiota departure from baseline. Volcano plot showing coefficients from penalized generalized estimating equations (PGEE) and their corresponding $p$ values. The model included clr abundances of baseline OTUs collapsed at genus level, decay averaged antibiotic histories, age, sex, body mass index, use of parenteral nutrition, baseline Shannon index, sample depth, and interval from baseline as potential predictors of the Aitchison distance between non-baseline microbiota and baseline (pre-treatment) microbiota for the same patient. Patient ID was a random effect. The tuning parameter was 0.21 , determined using fourfold cross validation. A first-order autoregressive correlation structure was assumed. The horizontal dashed line defines statistical significance $(p<0.05)$. Circles colored red or blue represent significant taxa with a coefficient $>10^{-3}$ (red) or $<-10^{-3}$ (blue), respectively. These taxa at baseline were associated with the extent of departure from baseline in subsequent samples and their corresponding circles were magnified for better visualization

genera), decay averaged antibiotic histories (7 features), age, sex, body mass index, use of parenteral nutrition, baseline Shannon index, sample read depth, and interval from baseline. Patient ID was a random effect. Fourfold cross validation determined the optimal value of the tuning parameter to be 0.21 . This value was then used to build the final PGEE model. In the final model, 5 baseline taxa (Roseburia, Blautia, Eggerthella, a Lachnospiraceae genus, and a Clostridiales genus) predicted larger $\Delta \mathrm{m}$ values, and one taxon (Bacteroides) predicted smaller $\Delta \mathrm{m}$ values (Fig. 4). None of the other variables predicted $\Delta \mathrm{m}$. In a case study to demonstrate these findings (Fig. 3d), we chose two subjects: (i) case 1 with a relatively high abundance of Bacteroides in the baseline sample (34\%) and (ii) case 2 with no Bacteroides in the baseline sample. As expected from PGEE results and with Bacteroides predicted to protect against change from baseline, subsequent samples from case 1 were less different from baseline (Aitchison distances 25.7-36.0) compared to subsequent samples from case 2 relative to baseline (Aitchison distances 30.2-56.8). This is also demonstrated in the PCA plot (Fig. 3b).

In post hoc exploratory analysis (Additional file 1 Table S1), we performed microfluidic quantitative PCR for antibiotic resistance genes (ARGs) in 27 longitudinal stool samples from 4 patients to understand whether obvious changes occur in longitudinal abundances of ARGs. The 3 most abundant ARGs were ermB (macrolide resistance, aggregate mean among samples: 6.57 copies/microL DNA), tetM (tetracycline resistance, 5.87 copies/microL DNA), and ermF (macrolide resistance, 5.30 copies/microL DNA). However, because antibiotics in these classes were not used in our patients, the ARGs likely did not play a role in our findings. vanA was the only ARG with clear dynamics in longitudinal samples. vanA confers vancomycin resistance to highly relevant pathogens in our patient population such as vancomycin-resistant enterococci. In two subjects, vanA 
was nearly or completely undetectable in the first week, then quickly rose in abundance in week 2 , and remained high until the end of hospitalization.

\section{Discussion}

We found for the first time that the pre-treatment state of human gut microbiota is a major determinant of antibiotic-induced microbiota perturbations. This finding in a clinical setting with intense antibiotic pressure suggests an even stronger predictive role for baseline microbiota when antibiotic exposure is less intense. Therefore, an important mechanism underlying individualized responses to antibiotics is the individualized microbiota at baseline. Considering that only a small fraction of gut microbiota is shared among healthy individuals $[38,39]$, our results explain the previous observations that the microbiota in different individuals responds differently to antibiotics $[2,40]$. This is also consistent with the role of pre-treatment microbiota in determining response to fecal microbiota transplantation (FMT) [41] and dietary interventions [42].

We identified 6 pre-treatment taxa predictive of the magnitude of microbiota perturbation from baseline. Roseburia, Blautia, and Eggerthella predicted larger microbiota departures from baseline. Specific Roseburia species degrade dietary fiber $\beta$-mannan, producing short-chain fatty acids such as butyrate, with numerous and profound homeostatic effects on the host [43]. Specific compounds synthesized by bacteria can regulate the transcription of various genes within the community (quorum sensing) [44], potentially modulating microbiota susceptibility to antibiotics. Similarly, certain Eggerthella species have significant metabolic potential, contributing, for example, to the conversion of dietary fiber-derived lignans to bioactive compounds [45]. Whether and how this metabolic activity can impact the gut microbiota is unknown. Antimicrobial peptides produced by certain Blautia species have been shown to confer colonization resistance against antibiotic-resistant pathogens [46]. A microbial community with fewer antibiotic-resistant genes is expected to undergo larger perturbations after exposure to antibiotics. Bacteroides was the only genus with an apparent stabilizing effect. Quorum sensing has been well established for some species of this genus, which are abundant in the colonic microbiota (e.g., Bacteroides fragilis). Examples of quorum sensing mechanisms that could contribute to differential response to antibiotics include autoinducers of efflux pumps and biofilm formation [47]. Bacteroides species can also influence microbiota composition by secreting antimicrobial peptides in contact-dependent and -independent manners [48]. In addition, Bacteroides species are potent producers of propionate [49], which mediates colonization resistance against pathogens such as Salmonella typhimurium [50].

Considering the two phases of microbiota response to antibiotics, our study focused on phase 1 , where the microbiota is perturbed under active antibiotic pressure. We included the characteristics of the antibiotic insult and baseline microbiota as the main potential determinants of phase 1 in our analysis. Although sex, age, and mode of nutrition were adjusted for, the role of other host factors such as genetics and immunity in phase 1 need to be evaluated in future research. Other potentially important variables include the specific type(s) of chemotherapy and proton pump inhibitors (PPIs). Chemotherapeutic regimens commonly used for patients with AML are not known to significantly alter gut microbiota, although an expansion of Enterococcus spp. and Escherichia coli has been reported [51]. In otherwise healthy adults and patients with inflammatory bowel disease, PPIs increased ectopic colonization of oral bacteria in the gut and promoted the expansion of Enterococcus, Streptococcus, Staphylococcus, and some Proteobacteria $[52,53]$. We use PPIs in our patients only if they develop significant upper gastrointestinal side effects from chemotherapy. In our experience, such patients typically require parenteral nutrition, a variable included in our models. To avoid anticipated multicollinearity, we did not consider PPI use as a separate variable. Studies with larger sample sizes would permit building more comprehensive models incorporating a larger number of variables. In addition, phase 2 (recovery) is a critical segment of the microbiota trajectory that needs to be studied further. Finally, how the two phases are interrelated and contribute to clinical outcomes is unknown. It is unclear, for example, whether robust microbiota recovery after perturbation towards baseline might compensate for a large initial departure from baseline. Also, whether a larger departure from baseline predicts a smaller chance for full recovery is unknown.

In conclusion, knowledge about pre-treatment microbiota can be used to predict the magnitude of antibioticinduced perturbations and lead to personalized precision therapeutics. We identified specific taxa in the pretreatment microbiota that may predict the extent of antibiotic perturbation. Our findings lead to hypothesis generation for testing and validation in future studies. Mechanisms by which specific taxa in gut microbiota communicate with other members of this complex community to regulate stability, resistance, and resilience is a subject of intense investigation. Quorum sensing and horizontal transfer of ARGs are two possible mechanisms. Our preliminary results on ARGs suggest potentially important ARGs with dynamics that could influence microbiota response to antibiotics over time. Finally, the patient population in this study was one with 
aggressive cancer receiving chemotherapy in a nosocomial setting and with altered nutrition. The extent to which our findings may be generalized to other clinical settings with antibiotic exposure remains to be determined.

\section{Supplementary Information}

The online version contains supplementary material available at https://doi. org/10.1186/s40168-021-01170-2.

Additional file 1: Figure S1 Taxonomic distribution in baseline and non-baseline samples. (a) Mean relative abundance of the most abundant taxa are shown. The top 15 taxa in each group were used to generate the plot. (b) Linear discriminant analysis Effect Size using an LDA score threshold of 3.0 and $p$ value threshold of 0.05 . The deepest level of taxonomy was genus (g), and taxa unclassifiable at the genus level are shown at family ( $f$ ) or order (o) level. Figure S2 Procrustes analysis correlating microbiota composition with antibiotic history. (a) Decay factor 2.0. (b) Decay factor 1.5. (c) Decay factor 1.1. Figure S3. Principal component mixed effect regression. The first PC of microbiota for the baseline sample, first PC of antibiotic history for the non-baseline sample, baseline Shannon diversity, read depth of the non-baseline sample, use of parenteral nutrition (categorical) before the non-baseline sample, and the time interval in days between the two samples were included as fixed effect predictors of Aitchison distance between the two samples. Patient ID was a random effect. Model performance was defined as the Pearson's correlation coefficient $(r)$ comparing observed vs. predicted values of the outcome variable. Different values of the decay factor were used in the two panels. PC: principal component. Table S1 Microfluidic quantitative PCR of antibiotic resistance genes.

\section{Acknowledgements}

We thank Dr. Satoshi Ishii for performing microfluidic quantitative PCR for antibiotic resistance genes and Michael Franklin for language editing.

\section{Authors' contributions}

A.R. and C.S. conceived, designed, and supervised the study. A.R., T.K., and C.S. performed microbiota analysis. M.E., H.E., and T.U.R. collected metadata. A.R. was responsible for the conduct of the clinical protocol. T.K., H.N., and C.S. extracted and sequenced DNA. A.R. wrote the manuscript. The authors read and approved the final manuscript.

\section{Funding}

This work was supported by the National Institutes of Health's National Center for Advancing Translational Sciences grants KL2TR002492 and UL1TR002494. The content is solely the responsibility of the authors and does not necessarily represent the official views of the National Institutes of Health's National Center for Advancing Translational Sciences.

\section{Availability of data and materials}

Sequence data were deposited in the Sequence Read Archive of the National Center for Biotechnology's Information (NCBI) under BioProject ID SRP141394.

\section{Declarations}

\section{Ethics approval and consent to participate}

The protocol was approved by the University of Minnesota Institutional Review Board. All patients provided written informed consent.

\section{Consent for publication}

$\mathrm{n} / \mathrm{a}$

\section{Competing interests}

The authors declare that they have no competing interests.

\section{Author details}

${ }^{1}$ Division of Hematology, Oncology, and Transplantation, Department of Medicine, University of Minnesota, 14-100 PWB, MMC 480, 420 Delaware St. SE, Minneapolis, MN 55455, USA. ²Division of Gastroenterology, Hepatology, and Nutrition, Department of Medicine, University of Minnesota, Minneapolis, MN, USA. ${ }^{3}$ Department of Surgery, University of Minnesota, Minneapolis, MN, USA.

Received: 2 June 2021 Accepted: 25 September 2021

Published online: 27 October 2021

\section{References}

1. Lozupone CA, Stombaugh JI, Gordon JI, Jansson JK, Knight R. Diversity, stability and resilience of the human gut microbiota. Nature. 2012;489:22030.

2. Dethlefsen L, Relman DA. Incomplete recovery and individualized responses of the human distal gut microbiota to repeated antibiotic perturbation. Proc Natl Acad Sci U S A. 2011;108(Suppl 1):4554-61.

3. Rashidi A, Kaiser T, Holtan SG, Weisdorf DJ, Khoruts A, Staley C. Pretransplant recovery of microbiome diversity without recovery of the original microbiome. Bone Marrow Transplant. 2019;54:1115-7.

4. May RM. Thresholds and breakpoints in ecosystems with a multiplicity of stable states. Nature. 1977;269:471-7.

5. Scheffer M, Carpenter S, Foley JA, Folke C, Walker B. Catastrophic shifts in ecosystems. Nature. 2001;413:591-6.

6. Ingrisch J, Bahn M. Towards a comparable quantification of resilience. Trends Ecol Evol. 2018;33:251-9.

7. Dogra SK, Doré J, Damak S. Gut microbiota resilience: definition, link to health and strategies for intervention. Front Microbiol. 2020;11:572921.

8. Sommer F, Anderson JM, Bharti R, Raes J, Rosenstiel P. The resilience of the intestinal microbiota influences health and disease. Nat Rev Microbiol. 2017; 15:630-8.

9. Bohnhoff M, Drake BL, Miller CP. Effect of streptomycin on susceptibility of intestinal tract to experimental salmonella infection. Proc Soc Exp Biol Med. 1954;86:132-7.

10. Kim S, Covington A, Pamer EG. The intestinal microbiota: antibiotics, colonization resistance, and enteric pathogens. Immunol Rev. 2017;279:90105.

11. Rowland I, Gibson G, Heinken A, Scott K, Swann J, Thiele I, et al. Gut microbiota functions: metabolism of nutrients and other food components. Eur J Nutr. 2018;57:1-24.

12. Zimmermann M, Zimmermann-Kogadeeva M, Wegmann R, Goodman AL. Mapping human microbiome drug metabolism by gut bacteria and their genes. Nature. 2019;570:462-7.

13. Martin AM, Sun EW, Rogers GB, Keating DJ. The influence of the gut microbiome on host metabolism through the regulation of gut hormone release. Front Physiol. 2019;10:428.

14. Relman DA. The human microbiome: ecosystem resilience and health. Nutr Rev. 2012;70(Suppl 1):S2-9.

15. Moltzau Anderson J, Lipinski S, Sommer F, Pan W-H, Boulard O, Rehman A, et al. NOD2 influences trajectories of intestinal microbiota recovery after antibiotic perturbation. Cell Mol Gastroenterol Hepatol. 2020;10:365-89.

16. Lavelle A, Hoffmann TW, Pham H-P, Langella P, Guédon E, Sokol H. Baseline microbiota composition modulates antibiotic-mediated effects on the gut microbiota and host. Microbiome. 2019;7:111.

17. Raymond F, Ouameur AA, Déraspe M, labal N, Gingras H, Dridi B, et al. The initial state of the human gut microbiome determines its reshaping by antibiotics. ISME J. 2016;10:707-20.

18. Bucaneve G, Micozzi A, Menichetti F, Martino P, Dionisi MS, Martinelli G, et al. Levofloxacin to prevent bacterial infection in patients with cancer and neutropenia. N Engl J Med. 2005;353:977-87.

19. Gohl DM, Vangay P, Garbe J, MacLean A, Hauge A, Becker A, et al. Systematic improvement of amplicon marker gene methods for increased accuracy in microbiome studies. Nat Biotechnol. 2016;34:942-9.

20. Bolyen E, Rideout JR, Dillon MR, Bokulich NA, Abnet CC, Al-Ghalith GA, et al. Reproducible, interactive, scalable and extensible microbiome data science using QIIME 2. Nat Biotechnol. 2019;37:852-7.

21. Al-Ghalith GA, Hillmann B, Ang K, Shields-Cutler R, Knights D. SHI7 is a selflearning pipeline for multipurpose short-read DNA quality control. mSystems. 2018;3 Available from: https://doi.org/10.1128/mSystems.00202-17. 
22. Magoč T, Salzberg SL. FLASH: fast length adjustment of short reads to improve genome assemblies. Bioinformatics. 2011;27:2957-63.

23. Al-Ghalith GA, Montassier E, Ward HN, Knights D. NINJA-OPS: fast accurate marker gene alignment using concatenated ribosomes. PLoS Comput Biol. 2016;12:e1004658

24. DeSantis TZ, Hugenholtz P, Larsen N, Rojas M, Brodie EL, Keller K, et al. Greengenes, a chimera-checked 165 rRNA gene database and workbench compatible with ARB. Appl Environ Microbiol. 2006;72:5069-72.

25. Langmead B, Salzberg SL. Fast gapped-read alignment with bowtie 2. Nat Methods. 2012;9:357-9.

26. Sandberg KD, Ishii S, LaPara TM. A microfluidic quantitative polymerase chain reaction method for the simultaneous analysis of dozens of antibiotic resistance and heavy metal resistance genes. Environ Sci Technol Lett. 2018; 5:20-5.

27. Ishii S, Segawa T, Okabe S. Simultaneous quantification of multiple foodand waterborne pathogens by use of microfluidic quantitative PCR [internet]. Appl Environ Microbiol. 2013:2891-8 Available from: https://doi. org/10.1128/aem.00205-13.

28. Segata N, Izard J, Waldron L, Gevers D, Miropolsky L, Garrett WS, et al. Metagenomic biomarker discovery and explanation. Genome Biol. 2011;12: R60.

29. Aitchison J, Barceló-Vidal C, Martín-Fernández JA, Pawlowsky-Glahn V. Logratio analysis and compositional distance. Math Geol. 2000;32:271-5.

30. Shannon CE, Weaver W. The mathematical theory of communication, vol. 11. Urbana: Univ. Illinois press; 1949. p. 117.

31. Anderson M, Gorley R, Clarke K, Anderson MJ, Gorley RN, Clarke KR, et al. PERMANOVA+ for PRIMER. Guide to software and statistical methods. 2008; Available from: https://www.scienceopen.com/document?vid=adfcaf4dc5cd-44a4-b1b7-f431e0410f4a

32. Gower JC. Generalized procrustes analysis. Psychometrika. 1975;40:33-51.

33. Inan G, Wang L. PGEE: an R package for analysis of longitudinal data with high-dimensional covariates. R J. 2017;9:393.

34. Wang L, Zhou J, Qu A. Penalized generalized estimating equations for highdimensional longitudinal data analysis [internet]. Biometrics. 2012. p. 353360. Available from: https://doi.org/10.1111/j.1541-0420.2011.01678.x

35. Liang K-Y, Zeger SL. Longitudinal data analysis using generalized linear models. Biometrika. 1986;73:13-22.

36. Rashidi A, Kaiser T, Shields-Cutler R, Graiziger C, Holtan SG, Rehman TU, et al. Dysbiosis patterns during re-induction/salvage versus induction chemotherapy for acute leukemia. Sci Rep. 2019;9:6083.

37. Rashidi A, Kaiser T, Graiziger C, Holtan SG, Rehman TU, Weisdorf DJ, et al. Gut dysbiosis during antileukemia chemotherapy versus allogeneic hematopoietic cell transplantation. Cancer. 2020;126:1434-47.

38. Falony G, Joossens M, Vieira-Silva S, Wang J, Darzi Y, Faust K, et al. Population-level analysis of gut microbiome variation. Science. 2016;352: 560-4.

39. Leónidas Cardoso L, Durão P, Amicone M, Gordo I. Dysbiosis individualizes the fitness effect of antibiotic resistance in the mammalian gut. Nat Ecol Evol. 2020;4:1268-78.

40. Dethlefsen $L$, Huse S, Sogin ML, Relman DA. The pervasive effects of an antibiotic on the human gut microbiota, as revealed by deep $16 \mathrm{~S}$ rRNA sequencing. PLoS Biology. 2008:e280 Available from: https://doi.org/10.13 71/journal.pbio.0060280.

41. Smillie CS, Sauk J, Gevers D, Friedman J, Sung J, Youngster I, et al. Strain Tracking reveals the determinants of bacterial engraftment in the human gut following fecal microbiota transplantation. Cell Host Microbe. 2018;23: 229-40.e5.

42. Zeevi D, Korem T, Zmora N, Israeli D, Rothschild D, Weinberger A, et al. Personalized nutrition by prediction of glycemic responses. Cell. 2015. p. 1079-1094. Available from: https://doi.org/10.1016/j.cell.2015.11.001

43. La Rosa SL, Leth ML, Michalak L, Hansen ME, Pudlo NA, Glowacki R, et al, The human gut Firmicute Roseburia intestinalis is a primary degrader of dietary $\beta$-mannans. Nat Commun. 2019;10:905.

44. Whiteley M, Diggle SP, Greenberg EP. Progress in and promise of bacterial quorum sensing research. Nature. 2017;551:313-20.

45. Bess EN, Bisanz JE, Yarza F, Bustion A, Rich BE, Li X, et al. Genetic basis for the cooperative bioactivation of plant lignans by Eggerthella lenta and other human gut bacteria. Nat Microbiol. 2020;5:56-66.

46. Kim SG, Becattini S, Moody TU, Shliaha PV, Littmann ER, Seok R, et al. Microbiota-derived lantibiotic restores resistance against vancomycinresistant enterococcus. Nature. 2019;572:665-9.
47. Pumbwe L, Skilbeck CA, Wexler HM. Presence of quorum-sensing systems associated with multidrug resistance and biofilm formation in Bacteroides fragilis. Microb Ecol. 2008;56:412-9.

48. Coyne MJ, Béchon N, Matano LM, McEneany VL, Chatzidaki-Livanis M, Comstock LE. A family of anti-Bacteroidales peptide toxins wide-spread in the human gut microbiota. Nat Commun. 2019;10:3460.

49. Reichardt N, Duncan SH, Young P, Belenguer A, McWilliam Leitch C, Scott $K P$, et al. Phylogenetic distribution of three pathways for propionate production within the human gut microbiota. ISME J. 2014;8:1323-35.

50. Jacobson A, Lam L, Rajendram M, Tamburini F, Honeycutt J, Pham T, et al. A gut commensal-produced metabolite mediates colonization resistance to salmonella infection. Cell Host Microbe. 2018;24:296-307.e7.

51. Hueso T, Ekpe K, Mayeur C, Gatse A, Joncquel-Chevallier Curt M, Gricourt G, et al. Impact and consequences of intensive chemotherapy on intestinal barrier and microbiota in acute myeloid leukemia: the role of mucosal strengthening. Gut Microbes. 2020;12:1800897.

52. Imhann F, Bonder MJ, Vich Vila A, Fu J, Mujagic Z, Vork L, et al. Proton pump inhibitors affect the gut microbiome. Gut. 2016;65:740-8.

53. Jackson MA, Goodrich JK, Maxan M-E, Freedberg DE, Abrams JA, Poole AC, et al. Proton pump inhibitors alter the composition of the gut microbiota. Gut. 2016;65:749-56.

\section{Publisher's Note}

Springer Nature remains neutral with regard to jurisdictional claims in published maps and institutional affiliations.
Ready to submit your research? Choose BMC and benefit from:

- fast, convenient online submission

- thorough peer review by experienced researchers in your field

- rapid publication on acceptance

- support for research data, including large and complex data types

- gold Open Access which fosters wider collaboration and increased citations

- maximum visibility for your research: over $100 \mathrm{M}$ website views per year

At BMC, research is always in progress.

Learn more biomedcentral.com/submissions 